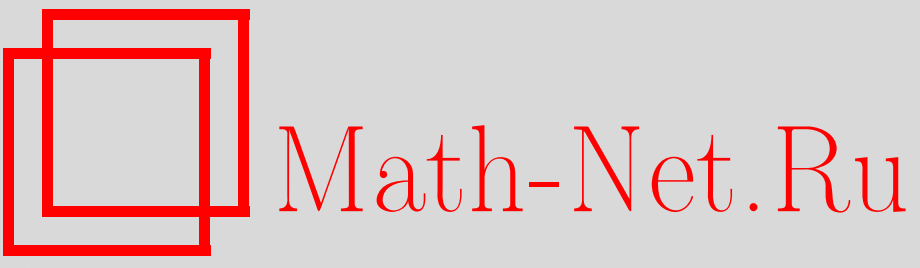

S. A. Nazarov, Trapping elastic waves by a semi-infinite cylinder with partly fixed surface, Sibirsk. Mat. Zh., 2020, Volume 61, Number 1, 160-174

DOI: https://doi.org/10.33048/smzh.2020.61.111

Use of the all-Russian mathematical portal Math-Net.Ru implies that you have read and agreed to these terms of use

http://www.mathnet.ru/eng/agreement

Download details:

IP : 35.173 .137 .237

April 26, 2023, 12:36:49 


\title{
ЗАХВАТ УПРУГИХ ВОЛН ПОЛУБЕСКОНЕЧНЫМ АНИЗОТРОПНЫМ ЦИЛИНДРОМ С ЧАСТИЧНО ЗАФИКСИРОВАННОЙ ПОВЕРХНОСТЬЮ
}

\author{
С. А. Назаров
}

\begin{abstract}
Аннотация. Рассматривается трехмерная смешанная краевая задача теории упругости о гармонических во времени колебаниях полубесконечного анизотропного цилиндра. Показано, что при определенных положении и форме зоны защемления поверхности происходит захват упругой волны, т. е. задача приобретает нетривиальное решение с экспоненциальным затуханием на бесконечности, или, наоборот, захваченной волны гарантированно нет на любой частоте колебаний. Сформулированы открытые вопросы о схожих спектральных задачах.
\end{abstract}

DOI 10.33048/smzh.2020.61.111

Ключевые слова: анизотропный цилиндрический волновод, система уравнений теории упругости, захваченные волны, собственные частоты.

1. Постановка задачи о цилиндрическом упругом волноводе. Пусть $\Omega=\omega \times \mathbb{R}_{+} \subset \mathbb{R}^{3}$ - полуцилиндр, сечение $\omega$ которого - область на плоскости $\mathbb{R}^{2}$, ограниченная замкнутым простым гладким (класса $C^{\infty}$ для простоты; ср. п. $\left.6,2^{0}\right)$ контуром $\partial \omega$. В двумерной окрестности контура введем систему криволинейных координат $(n, s)$, где $s$ - длина дуги на $\partial \omega$, а $n$ - ориентированное расстояние до $\partial \omega, n>0$ вне $\omega$. Декартовы координаты $x=\left(x_{1}, x_{2}, x_{3}\right)$ представим в виде $(y, z)$; здесь $z=x_{3} \in \mathbb{R}_{+}=(0,+\infty)$ и $y=\left(y_{1}, y_{2}\right) \in \omega$. Пусть еще $H$ - гладкая положительная функция переменной $s \in \partial \omega$ и (рис. $1(\mathrm{a}))$

$$
\Gamma_{H}=\{x \in \partial \Omega: 0<z<H(s)\}, \quad \omega^{0}=\omega \times\{0\}, \quad \Sigma_{H}=\{x \in \partial \Omega: z>H(s)\} .
$$

Рассмотрим задачу о гармонических во времени колебаниях упругого тела $\Omega$ с различными краевыми условиями на поверхностях (1), образующих границу тела. Уравнения теории упругости запишем в удобном для дальнейших выкладок матричном виде ${ }^{1)}$, а именно, вектор смещений $u$ интерпретируем как столбец $\left(u_{1}, u_{2}, u_{3}\right)^{\top}$, причем $\top-$ знак транспонирования и $u_{j}$ - проекция вектора на ось $x_{j}$. Составим столбец деформаций

$$
\varepsilon=\left(\varepsilon_{11}, \varepsilon_{22}, \varepsilon_{33}, \sqrt{2} \varepsilon_{23}, \sqrt{2} \varepsilon_{31}, \sqrt{2} \varepsilon_{12}\right)^{\top}
$$

из декартовых компонент тензора деформаций

$$
\varepsilon_{j, k}(u)=\frac{1}{2}\left(\frac{\partial u_{j}}{\partial x_{k}}+\frac{\partial U_{k}}{\partial x_{j}}\right), \quad j, k=1,2,3 .
$$

\footnotetext{
Работа выполнена за счет Российского научного фонда (проект 17-11-01003).

1) В англоязычной литературе она называется the Voigt-Mandel notation, но в русскоязычной связывается с именем С. Г. Лехницкого [1].
}

(c) 2020 Назаров C. A. 
Связь $\varepsilon(u)=D(\nabla) u$ столбцов деформаций и смещений включает матричный дифференциальный оператор первого порядка

$$
D(\nabla)=\left(\begin{array}{cccccc}
\partial_{1} & 0 & 0 & 0 & 2^{-1 / 2} \partial_{3} & 2^{-1 / 2} \partial_{2} \\
0 & \partial_{2} & 0 & 2^{-1 / 2} \partial_{3} & 0 & 2^{-1 / 2} \partial_{1} \\
0 & 0 & \partial_{3} & 2^{-1 / 2} \partial_{2} & 2^{-1 / 2} \partial_{1} & 0
\end{array}\right), \quad \nabla=\left(\begin{array}{c}
\partial_{1} \\
\partial_{2} \\
\partial_{3}
\end{array}\right)
$$

где $\partial_{j}=\frac{\partial}{\partial x_{j}}$ и множители $2^{1 / 2}$ и $2^{-1 / 2}$ введены в выражения (2) и (4) для того, чтобы уровнять натуральные нормы тензора и изображающего его столбца. Вектор напряжений $\sigma(u)$ аналогичного (2) строения находится согласно закону Гука

$$
\sigma(u ; x)=A \varepsilon(u ; x)=A D(\nabla) u(x),
$$

в котором $A-$ матрица жесткости, составленная для упругих модулей, имеющая размер $6 \times 6$ и являющаяся симметричной и положительно определенной. Тот факт, что $A-$ постоянная и произвольная матрица, означает однородность и анизотропность упругого материала волновода. Если материал изотропный, то

$$
A=\left(\begin{array}{ll}
A^{1} & \mathbb{O}_{3} \\
\mathbb{O}_{3} & A^{2}
\end{array}\right), \quad A^{1}=\left(\begin{array}{ccc}
\lambda+2 \mu & \lambda & \lambda \\
\lambda & \lambda+2 \mu & \lambda \\
\lambda & \lambda & \lambda+2 \mu
\end{array}\right), \quad A^{2}=\left(\begin{array}{ccc}
2 \mu & 0 & 0 \\
0 & 2 \mu & 0 \\
0 & 0 & 2 \mu
\end{array}\right) .
$$

Здесь $\mathbb{O}_{m}-$ нулевая матрица размером $m \times m$, а $\lambda \geq 0$ и $\mu>0-$ постоянные Ламе.

(a)

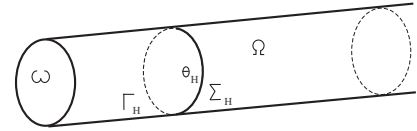

(b)

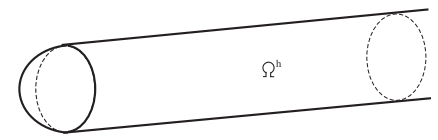

Рис. 1. Полубесконечные цилиндры с прямым (a) и искривленным (b) торцами.

Дифференциальные уравнения упругих колебаний тела $\Omega$ в матричном виде выглядят так:

$$
D(-\nabla)^{\top} A D(\nabla) u(x)=\Lambda u(x), \quad x \in \Omega .
$$

При этом $\Lambda=\rho \kappa^{2}-$ спектральный параметр, $\kappa>0-$ частота колебаний, а $\rho>0-$ постоянная плотность материала. Систему уравнений (6) снабдим краевыми условиями

$$
u(x)=0, x \in \Gamma_{H} \cup \omega^{0}, \quad D(\nu(x))^{\top} A D(\nabla) u(x)=0, x \in \Sigma_{H},
$$

или

$$
u(x)=0, x \in \Sigma_{H} \cup \omega^{0}, \quad D(\nu(x))^{\top} A D(\nabla) u(x)=0, x \in \Gamma_{H},
$$

где $\nu=\left(\nu_{1}, \nu_{2}, \nu_{3}\right)^{\top}$ - единичный вектор внешней нормали к поверхности $\partial \Omega$, определенный всюду, кроме ребра $\partial \omega^{0}=\partial \omega \times\{0\}$ на торце полуцилиндра $\Omega$, а матрица-функция $D(\nu)$ размером $6 \times 3$ получена заменами $\partial_{j} \mapsto \nu_{j}$ в формуле (4). Первое условие в (7) и (8) означает, что указанная часть поверхности 
жестко защемлена, а второе подразумевает отсутствие внешних нагрузок на остальной части границы.

Вариационная формулировка сформулированных краевых задач апеллирует к интегральному тождеству $[2,3]$

$$
(A D(\nabla) u, D(\nabla) v)_{\Omega}=\Lambda(u, v)_{\Omega} \quad \forall v \in \mathscr{H},
$$

в котором $(,)_{\Omega}$ - натуральное скалярное произведение в пространстве Лебега $L^{2}(\Omega)$, скалярном или векторном, а $\mathscr{H}=\mathscr{H}_{\Gamma}$ или $\mathscr{H}=\mathscr{H}_{\Sigma}-$ подпространства в пространстве Соболева $H^{1}(\Omega)^{3}$ (тройка указывает количество компонент у вектор-функции), выделенные условиями Дирихле в списках (7) или (8) соответственно. Поскольку левая часть $(9)$ - положительная симметричная и замкнутая в $H^{1}(\Omega)^{3}$ полуторалинейная форма, задачам $(6),(7)$ и $(6),(8)$ отвечают $\left[4\right.$, гл. 10] положительные самосопряженные операторы $\mathscr{A}_{\Gamma}$ и $\mathscr{A}_{\Sigma}$ соответственно. Из-за наличия линии

$$
\theta_{H}=\{x \in \partial \Omega: z=H(s)\}
$$

раздела краевых условий Дирихле и Неймана (ср. формулы (7), (8) и рис. 1(a)), на которой деформации и напряжения приобретают корневые особенности (см. далее п. 3), области определения упомянутых операторов содержат пространства $\mathscr{H}_{\Gamma}$ или $\mathscr{H}_{\Sigma}$, но заведомо не все вектор-функции из пространства Соболева $H^{2}(\Omega)^{3}$, удовлетворяющие условиям (7) или (8). Известно (см., например, [5]), что непрерывный спектр оператора $\mathscr{A}_{\Gamma}$ задачи (6), (7) занимает всю замкнутую положительную вещественную полуось $\overline{\mathbb{R}_{+}}=[0+\infty)$, а непрерывный спектр $\left[\Lambda_{\dagger},+\infty\right)$ оператора $\mathscr{A}_{\Sigma}$ имеет положительную точку отсечки $\Lambda_{\dagger}$.

Основная цель работы - убедиться в том, что при некоторых ограничениях на анизотропные свойства упругого материала точечный спектр оператора $\mathscr{A}_{\Gamma}$ пуст, т. е. у задачи (6), (7) нет собственных чисел, и привести пример захваченной упругой волны в задаче $(6),(8)$, а именно собственной вектор-функции оператора $\mathscr{A}_{\Sigma}$, принадлежащей пространству $H^{1}(\Omega)^{3}$ и потому экспоненциально затухающей на бесконечности.

2. Содержание работы. Известный классический прием [6] проверки отсутствия точечного спектра в задаче Дирихле для оператора Лапласа в квантовом цилиндрическом волноводе ${ }^{2)}$ (рис. 1(b))

$$
\Omega^{h}=\{x=(y, z): y \in \omega, z>h(y)\}, \quad h \in C^{1}(\bar{\omega}),
$$

был приспособлен [5] к смешанной краевой задаче теории для ортотропного тела упругости в случае условий Дирихле на искривленном торце $\omega^{h}=\{x: y \in$ $\omega, z=h(y)\}$ и, например, условий Неймана на боковой поверхности $\partial \Omega^{h} \backslash \overline{\omega^{h}}$ полуцилиндра (11) (ср. задачу $(6),(7)$ при $h=0)$. Этот прием подразумевает подстановку в формулу Грина собственной вектор-функции $u^{t r} \in H^{1}\left(\Omega^{h}\right)$ и ее производной $\partial_{z} u^{t r}$, а основную трудность в [5] составило доказательство включения $\partial_{z} u^{t r} \in H^{1}\left(\Omega^{h}\right)$ для произвольно анизотропного материала. В [5, $\S 2$, п. 3] установлено, что производные $\partial_{z} \varepsilon\left(u^{t r}\right)$ и $\partial_{z} \sigma\left(u^{t r}\right)$ остаются квадратично суммируемыми в окрестности ребра $\partial \gamma^{h} \subset \partial \Omega^{h}$ благодаря достаточно быстрому затуханию $O\left(r^{1+\delta}\right)$ поля смещений при $r \rightarrow+0$; здесь $\delta>0$ и $r$ - расстояние до ребра.

2) Вводимое ограничение на форму и гладкость торца можно ослабить. 


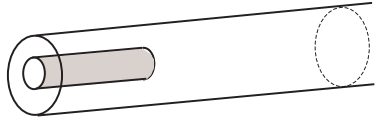

(b)

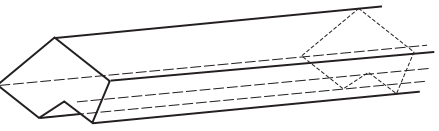

Рис. 2. Волновод с конечной цилиндрической трещиной (a) и волновод с ребристой поверхностью (b).

В $[7, \S 3$, п. 7] рассмотрен квантовый волновод (рис. 2(а))

$$
\Omega_{L}=\Omega \backslash\{x: y \in v, 0 \leq z \leq L\},
$$

где $v$ - простой замкнутый гладкий контур внутри сечения $\omega$, a $L>0$ - длина цилиндрической трещины, и показано, что задача Дирихле

$$
-\Delta u(x)=\lambda u(x), x \in \Omega_{L}, \quad u(x)=0, x \in \partial \Omega_{L},
$$

имеет собственное число (например, при большом $L$ ), несмотря на то, что производная $\partial_{z} u^{t r}$ удовлетворят задаче $(12)$ всюду, кроме торца $\omega^{0} \backslash v$, т. е. формально прием [6] работает. Такая неувязка объясняется анализом поведения собственной функции $u^{t r}$ около края $\{x: y \in v, z=L\}$ трещины: корневая сингулярность $O\left(r^{-1 / 2}\right)$ производной $\partial_{z} u^{t r}$ (см., например, [8, гл. 2]) выводит функцию $\partial_{z} u^{t r}$ из пространства Соболева $H^{1}\left(\Omega_{L}\right)$ и делает неправомочной подстановку пары $u^{t r}$ и $\partial_{z} u^{t r}$ в формулу Грина.

Из-за линии (10) смены типа краевых условий в задачах (6), (7) и (6), (8) мы сталкиваемся с аналогичной проблемой: производные решений этих задач также приобретают корневые сингулярности около линии $\theta_{H}$ (см. $[9,10]$ и далее лемму 1 в п. 3). Поэтому в п. 4 изменяем схему применения приема [6] и привлекаем формулу Грина в области

$$
\Omega(\rho)=\Omega \backslash \Theta_{\rho}, \quad \rho>0,
$$

где $r$ - расстояние до $\theta_{H}$, а $\Theta_{\rho}=\{x \in \Omega: r>\rho\}$ - трубчатая окрестность кривой $\theta_{H}$. Именно, проверяем, что предельный переход при $\rho \rightarrow+0$ в упомянутой формуле Грина обеспечивает соотношение

$$
\int_{\omega}{\overline{\partial z} u^{t r}(y, 0)}^{\top} A^{0} \partial_{z} u^{t r}(y, 0) d y \pm \int_{\theta_{H}} \widehat{\psi}(s) \sum_{\alpha=0, \pm}\left|K_{\alpha}(s)\right|^{2} d s=0,
$$

где черта означает комплексное сопряжение, $A^{0}$ - симметричная положительно определенная матрица, $\widehat{\psi}-$ положительная функция на $\theta_{H}$, а $K_{j}(s)-$ коэффициенты интенсивности напряжений (см. далее (42) и (45)). Важно то, что для задачи (6), (7) в правой части (14) реализуется знак плюс, поэтому

$$
u^{t r}(y, 0)=\partial_{z} u^{t r}(y, 0)=0 \quad \text { при } y=0 .
$$

Таким образом, теорема о единственности продолжения (см., например, [11, гл.4], а также обсуждение в $\left[5, \S 2\right.$, п. 1]) гарантирует, что $u^{t r}=0$ всюду в волноводе $\Omega$, т. е. предположение о существовании нетривиальной захваченной волны $u^{t r} \in H^{1}(\Omega)^{3}$ приводит к противоречию. Иными словами, точечный спектр задачи (6), (7) пуст. 
По-другому дело обстоит с задачей (6), (8), для которой равенство (6) приобретает знак минус и потому становится бесполезным. Более того, в п. 5 установим существование такого размера $l>0$, что в случае $H(s) \geq l, s \in \partial \omega$, задача (6), (8) приобретает захваченную упругую волну.

Полученные результаты допускают простую механическую интерпретацию. Краевые условия (7) отвечают удлиненной консоли, вделанной в неглубокое гнездо (обычный способ крепления). В этой ситуации захват волны невозможен. С другой стороны, краевые условия (8) означают, что полубесконечный штырь вставлен в отверстие в абсолютно жестком теле, но часть штыря выступает наружу. Если эта часть достаточно длинная, то она всегда производит захват одной или нескольких упругих волн.

3. Особенности упругих полей около линии смены типа краевых условий. В трехмерной окрестности контура (10) введем ортогональную систему криволинейных координат

$$
(\xi, \zeta)=\left(\xi_{1}, \xi_{2}, \zeta\right)=(n, \eta, \zeta)
$$

Ось $n$ направим вовне цилиндра $\Omega$ перпендикулярно его боковой поверхности, ось $\eta$ расположена в касательной к $\partial \omega \times \mathbb{R}$ плоскости перпендикулярно кривой $\theta_{H}$, а $\zeta$ - длина дуги на этой кривой. Действуя в соответствии с общей процедурой (см. $[10,11]$ и, например, гл. 8 в книге [8]) исследования асимптотики решений эллиптической краевой задачи около гладкого ребра на границе области, зафиксируем какую-либо точку $\zeta \in \theta_{H}$, перейдем в задаче $(6),(7)$ к координатам (15), заморозим коэффициенты в $\zeta^{\bullet}$ и полностью устраним производную $\partial_{\zeta}=\partial / \partial \zeta$. В результате придем к такой модельной задаче на нижней полуплоскости $\mathbb{R}_{-}^{2}=\left\{\xi: \xi_{1}<0\right\}$ :

$$
\begin{gathered}
L^{\bullet}\left(\nabla_{\xi}\right) U(\xi):=D\left(\nabla_{\xi}, 0\right)^{\top} A^{\bullet}\left(\zeta^{\bullet}\right) D\left(\nabla_{\xi}, 0\right) U(\xi)=0, \quad \xi \in \mathbb{R}_{-}^{2}, \\
U\left(0, \xi_{2}\right)=0, \quad \xi_{2}<0, \\
B^{\bullet}\left(\nabla_{\xi}\right) U\left(0, \xi_{2}\right):=D(-1,0,0)^{\top} A^{\bullet}\left(\zeta^{\bullet}\right) D\left(\nabla_{\xi}\right) U\left(0, \xi_{2}\right)=0, \quad \xi_{2}>0 .
\end{gathered}
$$

Приведем пояснения. Во-первых, ортогональное преобразование декартовых координат не влияет на общее строение дифференциальных операторов краевых задач теории упругости, но лишь изменяет матрицу жесткости, впрочем в изотропном случае матрицы (5) сохраняются полностью. Во-вторых, поскольку координаты $(\xi, \zeta)$ криволинейные, в системе уравнений (6) возникает дополнительное слагаемое - дифференциальный оператор первого порядка, а в краевом условии (7) на $\Sigma_{H}$ - оператор умножения на матрицу-функцию, однако такие возмущения младших порядков вместе со слагаемым $\Lambda u$ из правой части (6) устраняются посредством замораживания коэффициентов. В-третьих, система (16), изначально справедливая при малых $n=\xi_{1}<0$ и $\eta=\xi_{2}>0$, распространяется на всю полуплоскость $\mathbb{R}_{-}^{2}$, а краевые условия (17) и (18) - на полуоси, отрицательную и положительную соответственно. Наконец, ищутся так называемые степенно-логарифмические решения

$$
U(\xi)=r^{t} \Phi(\varphi, \ln r)
$$

задачи (16)-(18); при этом $(r, \varphi) \in \mathbb{R}_{+} \times(-\pi, 0)$ - система полярных координат на полупространстве $\mathbb{R}_{-}^{2}$, а комплексное число $t \in \mathbb{C}$ и вектор-функция $\Phi$, зависящая гладко от $\varphi \in[-\pi, 0]$ и полиномиально от $\ln r$, подлежат определению. 
Ввиду представлений операторов из левых частей (16) и (18)

$$
L^{\bullet}\left(\nabla_{\xi}\right)=r^{-2} \mathfrak{L}\left(\varphi, \partial_{\varphi}, r \partial_{r}\right), \quad B^{\bullet}\left(\nabla_{\xi}\right)=r^{-1} \mathfrak{B}\left(\partial_{\varphi}, r \partial_{r}\right)
$$

подстановка вектор-функции $r^{t} \Phi(\varphi)$ в задачу (16)-(18) порождает квадратичный пучок

$$
\begin{aligned}
t \mapsto \mathfrak{A}(t) \Phi=\left\{\mathfrak{L}\left(\varphi, \partial_{\varphi}, t\right) \Phi,\left.\Phi\right|_{\varphi=-\pi},\right. & \left.\left.\mathfrak{B}\left(\partial_{\varphi}, t\right) \Phi\right|_{\varphi=0}\right\} \\
: & H^{2}(-\pi, 0)^{3} \rightarrow L^{2}(-\pi, 0)^{3} \times \mathbb{C}^{3} \times \mathbb{C}^{3},
\end{aligned}
$$

т. е. $t-$ собственное число, а $\Phi-$ собственный вектор этого пучка.

Благодаря элементарной геометрии и простой структуре дифференциальных операторов с постоянными коэффициентами известна полная информация о спектре пучка (21), а именно, спектр состоит из чисел

$$
\frac{1}{2}+k, \frac{1}{2}+k \pm i \varkappa\left(\zeta^{\bullet}\right), \quad \text { где } k=0, \pm 1, \pm 2, \ldots
$$

Здесь $\varkappa\left(\zeta^{\bullet}\right)$ - величина, зависящая от матрицы $A^{\bullet}\left(\zeta^{\bullet}\right)$. Все собственные числа из списка (2) простые алгебраически, т. е. у собственных векторов пучка нет присоединенных векторов и в решениях (19) наверняка отсутствует зависимость от логарифма $\ln r$. Наконец, при $\varkappa\left(\zeta^{\bullet}\right)>0$ все собственные числа $(22)$ простые геометрически (единственный собственный вектор), но при $\varkappa\left(\zeta^{\bullet}\right)=0$ кратность каждого равна трем.

Приведенные факты устанавливаются по единой схеме, разработанной в $[9,10]$ для задач теории упругости о трещинах и распространенной в книге $[8$, гл. $7, \S 4]$ (см. также обзор $[12, \S 2$, п. 6 , предложение 2.22]) на широкий класс общих эллиптических краевых задач. Эта схема использует дифференцирование вдоль границы $\partial \mathbb{R}_{-}^{2}$ : производная $\partial_{\eta} U(\xi)$ решения $(19)$ удовлетворяет тем же равенствам (16)-(18) (см. ниже). Кроме того, в нашем конкретном случае применяется следующая простая алгебраическая лемма.

Лемма 1. Для вектор-функции

$$
U(\xi)=\left|\xi_{1}\right|^{t} a, \quad a \in \mathbb{C}^{3} \backslash\{0\},
$$

при $t=0$ и $t=1$ не выполнены краевые условия (17) и (18) соответственно. $B$ случае $t \neq 0,1$ она не удовлетворяет системе уравнений (16).

ДокАЗАТЕЛЬство. Имеем

$$
L^{\bullet}\left(\nabla_{\xi}\right) U(\xi)=-t(t-1)\left|\xi_{1}\right|^{t-2} \mathbf{A} a, \quad B^{\bullet}\left(\nabla_{\xi}\right) U(\xi)=-t\left|\xi_{1}\right|^{t-1} \mathbf{A} a .
$$

Поскольку $(3 \times 3)$-матрица $\mathbf{A}=D(1,0,0)^{\top} A^{\bullet}\left(\zeta^{0}\right) D(1,0,0)$ симметричная и положительно определенная, при $t \neq 0$ оба выражения (23) обращаются в нуль лишь при тривиальном столбце $a$. Если $t=0$, то $U(\xi)=a$ и $a=0$ в силу условия Дирихле (17).

Три степенных решения с показателями (22) при $k=0$

$$
U^{0}(\xi)=r^{1 / 2} \Phi^{0}(\varphi), \quad U^{ \pm}(\xi)=r^{ \pm i \varkappa\left(\zeta^{\bullet}\right)+1 / 2} \Phi^{ \pm}(\varphi)
$$

и их производные

$$
V^{0}(\xi)=\partial_{\eta} U^{0}(\xi)=r^{-1 / 2} \Psi^{0}(\varphi), \quad V^{ \pm}(\xi)=\partial_{\eta} U^{ \pm}(\xi)=r^{ \pm i \varkappa\left(\zeta^{\bullet}\right)-1 / 2} \Psi^{ \pm}(\varphi),
$$


имеющие вид (22) при $k=-1$, обладают специальным свойством, сыгравшим важную роль при доказательстве основной теоремы 2 , а именно вектор-функции (24) можно зафиксировать так, чтобы стали верными соотношения

$$
Q_{\rho}\left(U^{\alpha}, V^{\beta}\right)=\delta_{\alpha, \beta}, \quad \alpha, \beta=0, \pm .
$$

При этом $\delta_{\alpha, \beta}-$ символ Кронекера, а левая часть $(26)$ - симплектическая (полуторалинейная и антиэрмитова) форма

$$
\begin{aligned}
& Q_{\rho}(U, V)=\rho \int_{-\pi}^{0}\left(\overline{V(\xi)}^{\top} D(\cos \varphi, \sin \varphi, 0)^{\top} A^{\bullet}\left(\zeta^{\bullet}\right) D\left(\nabla_{\xi}, 0\right) U(\xi)\right. \\
&\left.\quad-U(\xi)^{\top} D(\cos \varphi, \sin \varphi, 0)^{\top} A^{\bullet}\left(\zeta^{\bullet}\right) D\left(\nabla_{\xi}, 0\right) \overline{V(\xi)}\right)\left.\right|_{r=\rho} d \varphi .
\end{aligned}
$$

Подынтегральное выражение из (27) включает вектор радиальных напряжений, поскольку $(\cos \varphi, \sin \varphi)^{\top}-$ нормаль к окружности. Таким образом, сама форма $(27)$ проистекает от формулы Грина для оператора $L^{\bullet}\left(\nabla_{\xi}\right)$ и потому не зависит от радиуса $\rho>0$ для всех степенных решений и их линейных комбинаций.

Для того чтобы обеспечить и проверить равенства (26), рассмотрим вспомогательную задачу

$$
\begin{gathered}
L^{\bullet}\left(\nabla_{\xi}\right) \mathscr{U}^{h}(\xi)=\mathscr{F}(\xi):=\left[L^{\bullet}\left(\nabla_{\xi}\right), \mathscr{X}\right] \mathscr{U}^{0}(\xi), \quad \xi \in \mathbb{R}_{-}^{2}, \\
\mathscr{U}^{h}\left(0, \xi_{2}\right)=0, \quad \xi_{2}<-h, \\
B^{\bullet}\left(\nabla_{\xi}\right) \mathscr{U}^{h}\left(0, \xi_{2}\right)=\mathscr{G}\left(\xi_{2}\right):=\left[B^{\bullet}\left(\nabla_{\xi}\right), \mathscr{X}\right] \mathscr{U}^{0}\left(0, \xi_{2}\right), \quad \xi_{2}>-h .
\end{gathered}
$$

Здесь $h>0$ - малый параметр, в правых частях фигурируют коммутаторы дифференциальных операторов $(20)$ с гладкой срезающей функцией $\mathscr{X}$,

$$
\mathscr{X}(\xi)=1 \text { при } r<1, \quad \mathscr{X}(\xi)=0 \text { при } r>2
$$

и, наконец,

$$
\mathscr{U}^{0}(\xi)=\sum_{\alpha=0, \pm} K_{\alpha} U^{\alpha}(\xi), \quad K_{\alpha} \in \mathbb{C} .
$$

Правые части (28) и (30) обращаются в нуль при $r<1$ и $r>2$, а значит, благодаря условию Дирихле (29), обеспечивающему неравенство Пуанкаре на дуге $(-\pi, 0) \ni \varphi$, задача $(28)-(30)$ имеет единственное решение $\mathscr{U}^{h} \in \mathscr{H}^{h}$, где $\mathscr{H}^{h}$ - весовое пространство Соболева вектор-функций, аннулирующихся на луче $\Upsilon^{h}=(-\infty,-h)$ и обладающих конечной нормой

$$
\left\|\mathscr{U}^{h} ; \mathscr{H}^{h}\right\|=\left(\left\|\nabla_{\xi} \mathscr{U}^{h} ; L^{2}\left(\mathbb{R}_{-}^{2}\right)\right\|^{2}+\left\|(1+r)^{-1} \mathscr{U}^{h} ; L^{2}\left(\mathbb{R}_{-}^{2}\right)\right\|^{2}\right)^{1 / 2}
$$

(ср. [13; 8, гл. 6] и, например, [9]). Более того, согласно [14] это решение доставляет минимум функционалу энергии

$$
\begin{aligned}
\mathscr{E}\left(\mathscr{U}^{h}\right)=\frac{1}{2}\left(A^{\bullet} D\left(\nabla_{\xi}\right) \mathscr{U}^{h},\right. & \left.D\left(\nabla_{\xi}\right) \mathscr{U}^{h}\right)_{\mathbb{R}_{-}^{2}}-\left(\mathscr{F}, \mathscr{U}^{h}\right)_{\mathbb{R}_{-}^{2}} \\
& -\left(\mathscr{G}, \mathscr{U}^{h}\right)_{\Upsilon h}=-\frac{1}{2}\left(\mathscr{F}, \mathscr{U}^{h}\right)_{\mathbb{R}_{-}^{2}}-\frac{1}{2}\left(\mathscr{G}, \mathscr{U}^{h}\right)_{\Upsilon h},
\end{aligned}
$$

причем последнее равенство получено интегрированием по частям при учете соотношений (28)-(30). Поскольку пространство $\mathscr{H}^{h}$ увеличивается при росте параметра $h$ (сужается область $\Upsilon^{h}$ постановки условия Дирихле), функция $h \mapsto$ $\mathscr{E}\left(\mathscr{U}^{h}\right)$ монотонно убывает. 
Вычислим приращение функционала (32) при малом $h$, построив асимптотику решения $\mathscr{U}^{h}$ при помощи метода сращиваемых асимптотических разложений (см., например, $[15,16])$, в интерпретации $[9,10]$, так как из-за сдвига точки $\mathscr{O}^{h}=(0,-h)$ смены типа краевых условий задачу $(28)-(30)$ следует интерпретировать как сингулярно возмущенную. В качестве главного члена внешнего разложения

$$
\mathscr{U}^{h}(\xi)=\mathscr{X}(\xi) \mathscr{U}^{0}(\xi)+h \mathscr{U}^{\prime}(\xi)+\ldots,
$$

справедливого на удалении от $\mathscr{O}^{h}$, возьмем линейную комбинацию из (31). Главный член внутреннего разложения

$$
\mathscr{U}^{h}(\xi)=\mathscr{W}^{0}\left(\xi^{h}\right)+\cdots=h^{1 / 2} K_{0} U^{0}\left(\xi^{h}\right)+\sum_{ \pm} h^{ \pm i \varkappa\left(\zeta^{\bullet}\right)+1 / 2} K_{ \pm} U^{ \pm}\left(\xi^{h}\right)+\ldots
$$

пригодного в непосредственной близости от точки $\mathscr{O}^{h}$, записан в растянутых координатах $\xi^{h}=\left(h^{-1}\left(\xi_{1}+h\right), h^{-1} \xi_{2}\right)$. Перейдем в $(34)$ к исходным координатам $\xi$ и при помощи соотношений $(24)$, а также формулы Тейлора относительно параметра $h$ получим

$$
\begin{aligned}
& \mathscr{W}^{0}\left(\xi^{h}\right)=\sum_{\alpha=0, \pm} K_{\alpha} U^{\alpha}\left(\xi_{1}+h, \xi_{2}\right) \\
&=\sum_{\alpha=0, \pm} K_{\alpha} U^{\alpha}\left(\xi_{1}, \xi_{2}\right)+h \sum_{\alpha=0, \pm} K_{\alpha} \partial_{\eta} U^{\alpha}\left(\xi_{1}, \xi_{2}\right)+\ldots
\end{aligned}
$$

Применим процедуру сращивания (см. $[15,16]$ и др.) внутреннего и внешнего разложений. Сразу заметим, что первый член в правой части (35) совпадает с линейной комбинацией (31), присутствующей в (33) в качестве главного члена. Кроме того, поправочный член во внешнем разложении принимает вид

$$
\mathscr{U}^{\prime}(\xi)=\sum_{\alpha=0, \pm} K_{\alpha} \partial_{\eta} U^{\alpha}(\xi)=\sum_{\alpha=0, \pm} K_{\alpha} V^{\alpha}(\xi)
$$

удовлетворяя задаче $(16)-(18)$ и затухая на бесконечности как $O\left(|\xi|^{-1 / 2}\right)$ согласно соотношениям (25).

Обоснование построенной асимптотики представлено в $[9,10 ; 8$, гл. $7, \S 4]$ и основано на общих результатах из $[17$, гл. 4,5$]$. В частности,

$$
\left|\mathscr{U}^{h}(\xi)-\mathscr{X}(\xi) \mathscr{U}^{0}(\xi)-h \mathscr{U}^{\prime}(\xi)\right| \leq c h^{2} \quad \text { при } \xi \in \overline{\mathbb{R}_{-}^{2}}, 1 \leq|\xi| \leq 2 .
$$

Благодаря расположению носителей правых частей $\mathscr{F}$ и $\mathscr{G}$ задачи (28)-(30) неравенство (36) позволяет вычислить величину (32):

$$
\begin{aligned}
&-\frac{1}{2}\left(\left(\mathscr{F}, \mathscr{U}^{h}\right)_{\mathbb{R}_{-}^{2}}+\right.\left.\left(\mathscr{G}, \mathscr{U}^{h}\right)_{\Upsilon^{h}}\right)=-\frac{1}{2}\left(\left(\mathscr{F}, \mathscr{X} \mathscr{U}^{0}\right)_{\mathbb{R}_{-}^{2}}+\left(\mathscr{G}, \mathscr{X} \mathscr{U}^{0}\right)_{\Upsilon^{0}}\right) \\
&-\frac{h}{2}\left(\left(\mathscr{F}, \mathscr{U}^{\prime}\right)_{\mathbb{R}_{-}^{2}}+\left(\mathscr{G}, \mathscr{U}^{\prime}\right)_{\Upsilon^{0}}\right)+O\left(h^{2}\right) \\
&= \mathscr{E}\left(\mathscr{X} \mathscr{U}^{0}\right)-\frac{h}{2} \sum_{\alpha, \beta=0, \pm} \overline{K_{\beta}} M_{\alpha \beta} K_{\alpha}+O\left(h^{2}\right) \\
&=\mathscr{E}\left(\mathscr{X} \mathscr{U}^{0}\right)-\frac{h}{2} \bar{K}^{\top} M K+O\left(h^{2}\right) .
\end{aligned}
$$

При этом $K=\left(K_{0}, K_{ \pm}, K_{-}\right)^{\top}-$ столбец коэффициентов интенсивности напряжений, а элементы $(3 \times 3)$-матрицы $M$ находятся при помощи формулы Грина 
в полуплоскости с вырезанным кругом $\mathbb{B}_{\rho}=\{\xi: r<\rho\}$, т. е.

$$
\begin{aligned}
M_{\alpha \beta}= & \left(L^{\bullet}\left(\nabla_{\xi}\right)\left(\mathscr{X} U^{\alpha}\right), V^{\beta}\right)_{\mathbb{R}_{-}^{2}}+\left(B^{\bullet}\left(\nabla_{\xi}\right)\left(\mathscr{X} U^{\alpha}\right), V^{\beta}\right)_{\Upsilon^{0}} \\
= & \lim _{\rho \rightarrow+0}\left(\left(L^{\bullet}\left(\nabla_{\xi}\right)\left(\mathscr{X} U^{\alpha}\right), V^{\beta}\right)_{\mathbb{R}_{-}^{2} \backslash \mathbb{B}_{\rho}}+\left(B^{\bullet}\left(\nabla_{\xi}\right)\left(\mathscr{X} U^{\alpha}\right), V^{\beta}\right)_{\Upsilon 0} \backslash \mathbb{B}_{\rho}\right) \\
& =\lim _{\rho \rightarrow+0} Q_{\rho}\left(U^{\alpha}, V^{\beta}\right) .
\end{aligned}
$$

Здесь учтено, что вектор $(\cos \varphi, \sin \varphi)$ направлен внутрь области $\mathbb{R}_{-}^{2} \backslash \overline{\mathbb{B}_{\rho}}$.

Поскольку функция

$$
h \mapsto \mathscr{E}\left(\mathscr{U}^{h}\right)-\mathscr{E}\left(\mathscr{X} \mathscr{U}^{0}\right)=-\frac{h}{2} \bar{K}^{\top} M K+O\left(h^{2}\right)
$$

строго монотонно убывает при любых коэффициентах $K \in \mathbb{C}^{3} \backslash\{0\}$, матрица $M$ должна быть симметричной и положительно определенной. Действительно, согласно общим результатам из [18] (см. также [8, гл. 3]) матрица с элементами $Q_{\rho}\left(U^{\alpha}, V^{\beta}\right)$ невырожденна, а значит, при отсутствии названных свойств можно подобрать коэффициенты $K_{\alpha}$ так, чтобы правая часть (38) стала положительной.

Если $\varkappa\left(\zeta^{\bullet}\right)>0$ в формулах $(22)$, то матрица $M$ диагональная, так как при $\alpha \neq \beta$ интеграл (27) с $U=U^{\alpha}$ и $V=V^{\beta}$ включает осциллирующий множитель $e^{ \pm i k \varkappa\left(\zeta^{\bullet}\right) \rho}$ при $k=1$ или $k=2$ и потому, имея конечный предел, обязан обратиться в нуль. В результате замены $U^{\alpha} \mapsto M_{\alpha \alpha}^{-1 / 2} U^{\alpha}$ матрица $M$ переделывается в единичную матрицу $\mathbb{I}_{3}$ размером $3 \times 3$. В случае $\varkappa\left(\zeta^{\bullet}\right)=0$ матрица $M$ может быть заполненной, однако равенство $M=\mathbb{I}_{3}$ достигается преобразованием базиса (24) при помощи матрицы $M^{-1 / 2}$ (обратная матрица для квадратного корня из симметричной и положительно определенной матрицы $M)$.

Напомним пропущенный в представленном анализе шаг схемы $[9 ; 8, \S 4$, п. 7], приводящей к формуле (22) для спектра пучка (21). Предположим, что у пучка есть собственное число $t$, для которого $\operatorname{Im} t \in(0,1 / 2) \cup(1 / 2,1)$. Согласно общим результатам работы [20] найдутся степенно-логарифмические решения $U^{t}$ и $V^{-\bar{t}}$, подчиненные требованию

$$
Q_{\rho}\left(U^{t}, V^{-\bar{t}}\right)=1
$$

В качестве верхних индексов вступают показатели однородности решений. Пусть еще $V^{t-1}$ и $V^{1-\bar{t}}$ - другие степенно-логарифмические решения, причем

$$
V^{t-1}=\partial_{\eta} U^{t}, \quad \partial_{\eta} U^{1-\bar{t}}=V^{-t}, \quad U^{t} \neq U^{1-\bar{t}} .
$$

Справедливы формулы

$$
\begin{aligned}
& 1=Q_{\rho}\left(U^{t}, \partial_{\eta} U^{1-\bar{t}}\right)=-Q_{\rho}\left(\partial_{\eta} U^{t}, U^{1-\bar{t}}\right)=\overline{Q_{\rho}\left(U^{1-\bar{t}}, \partial_{\eta} U^{t}\right)} \\
&=\overline{Q_{\rho}\left(U^{1-\bar{t}}, V^{t-1}\right)} \quad \Rightarrow \quad Q_{\rho}\left(U^{1-\bar{t}}, V^{t-1}\right)=1 .
\end{aligned}
$$

Здесь приняты во внимание равенства

$$
Q_{\rho}(V, U)=-\overline{Q_{\rho}(U, V)}, \quad Q_{\rho}\left(\partial_{\eta} U, V\right)=-Q_{\rho}\left(U, \partial_{\eta} V\right) ;
$$

первое - общее свойство симплектических форм, а второе, напоминающее интегрирование по частям, установлено для решений задачи $(16)-(18)$ в [9, п. 4; $12, \S 2$, п. 6$]$. 
Построим асимптотику решений задачи (28)-(30) с правыми частями, порожденными суммой

$$
\mathscr{U}^{0}(\xi)=K_{t} U^{t}(\xi)+K_{1-\bar{t}} U^{1-\bar{t}}(\xi)
$$

и при помощи равенств (39) и (40) получим аналогичное выкладке (37) соотношение

$$
\mathscr{E}\left(\mathscr{U}^{h}\right)-\mathscr{E}\left(\mathscr{U}^{0}\right)=-\frac{h}{2}\left(K_{t} \overline{K_{1-\bar{t}}}+K_{1-\bar{t}} \bar{K}_{t}\right),
$$

которое вступает в противоречие со свойством монотонности функции $h \longmapsto$ $\mathscr{E}\left(\mathscr{U}^{h}\right)$, так как коэффициенты $K_{t}$ и $K_{1-\bar{t}}$ линейной комбинации (41) выбираются независимо.

Точно так же проверяется отсутствие зависимости от $\ln r$ у решений (19) с показателями (22). Еще раз укажем, что более подробное изложение схемы можно найти в $[9,10 ; 12, \S 2]$.

4. Отсутствие захваченных волн. Пусть $u^{t r} \in \mathscr{H}-$ решение задачи $(6),(7)$ в вариационной постановке (9). Согласно общим результатам $[19,20]$ (см. также [8, гл. 8] и др. публикации), конкретизированным в п. 3, векторфункция $u^{t r}$, гладкая всюду, кроме линий $\partial \omega^{0}$ и $\theta_{H}$, на границе $\partial \Omega$, допускает представление

$$
u^{t r}(x)=\tilde{u}^{t r}(x)+\chi(x) \sum_{\alpha=0, \pm} K_{\alpha}(\zeta) U^{\alpha}(\zeta ; n, \eta)
$$

Здесь $\chi-$ гладкая срезающая функция,

$$
\chi(x)=1 \text { при } r<d / 2, \quad \chi(x)=0 \text { при } r>d,
$$

где $d>0$ - малое число, а $U^{\alpha}(\zeta ; n, \eta)$ - степенные решения (19) задачи (16)-(18) при $\zeta^{\bullet}=\zeta$ с показателями $(16), k=0$, причем зависимости $\theta_{H} \ni \zeta \mapsto U^{\alpha}(\zeta ; n, \eta)$ и $\zeta \mapsto K_{\alpha}(\zeta)$ можно считать гладкими (последние величины в теории упругости называются коэффициентами интенсивности напряжений). Наконец, для остатка $\tilde{u}^{t r}$ верны неравенства

$$
\left|\nabla_{\xi}^{p} \tilde{u}^{t r}(x)\right| \leq c_{\delta} r^{-\delta-p+3 / 2}, \quad p=0,1,2,
$$

где $\delta$ - любое положительное число, а множитель $c_{\delta}$ зависит от $\delta$, но не от $r \in(0, d]$.

Прием Рэллиха [6] подразумевает подстановку в формулу Грина векторфункций $u^{t r}$ и $\partial_{z} u^{t r}$. Как проверено в [5, $\S 2$, п. 3], сингулярности упругих полей на ребре $\partial \omega^{0}$ торца полуцилиндра не вызывают расходимость возникающих объемных и поверхностных интегралов. Вместе с тем корневые сингулярности производной $\partial_{z} u^{t r}$ поля (42) выводят ее из класса $H^{1}(\Omega)^{3}$, что препятствует непосредственному обобщению подхода [6].

Вырежем трубчатую окрестность контура (10) и применим формулу Грина в области (13). В результате получаем равенство

$$
\begin{aligned}
& \int_{\omega}{\overline{\partial_{z} u^{t r}(y, 0)}}^{\top} D(0,0,1)^{\top} A D\left(\nabla_{x}\right) u^{t r}(y, 0) d y \\
& =\int_{\partial \Omega(\rho) \backslash \partial \Omega}\left(\overline{\partial_{z} u^{t r}(x)} D(n(x))^{\top} A D\left(\nabla_{x}\right) u^{t r}(x)\right. \\
& \left.-u^{t r}(x) D(n(x))^{\top} A D\left(\nabla_{x}\right) \overline{\partial_{z} u^{\operatorname{tr}}(x)}\right) d s_{x},
\end{aligned}
$$


где $n$ - внешняя нормаль, а $d s_{x}$ - элемент площади поверхности вырезанной искривленной трубки $\Theta_{\rho}$.

При учете условия Дирихле на торце $\omega^{0}$ левая часть (43) преобразуется так же, как в $[5, \S 2$, п. 2], и приводится к виду

$$
\begin{aligned}
& \int_{\omega}{\overline{\partial_{z} u^{t r}(y, 0)}}^{\top} A^{0} \partial_{z} u^{t r}(y, 0) d y \geq c_{0}\left\|\partial_{z} u^{t r} ; L^{2}\left(\omega^{0}\right)\right\|^{2}, \\
& A^{0}=D(0,0,1)^{\top} A D(0,0,1)>0
\end{aligned}
$$

(ср. формулу (14)). Обработаем правую часть.

Заметим, что дифференцирование разложения (42) приводит к соотношению

$$
\partial_{z} u^{t r}(x)=\tilde{v}^{t r}(x)+\chi(x) \cos \psi(\zeta) \sum_{\alpha=0, \pm} K_{\alpha}(\zeta) \partial_{\eta} U^{\alpha}(\zeta ; \eta, n) .
$$

При этом $\psi(\zeta) \in(-\pi / 2, \pi / 2)-$ угол между осями $z$ и $\eta$, а последнее дифференцирование в представлении

$$
\partial_{z}=\cos \psi(\zeta) \partial_{\eta}-\sin \psi(\zeta) \partial_{\xi}
$$

не влияет на выделенные в (45) сингулярности $O\left(r^{-1 / 2}\right)$ благодаря гладкой зависимости ингредиентов от переменной $\zeta \in \theta_{H}$.

Учитывая разнонаправленность нормалей в формулах (27) и (43), устраняем в правой части последней бесконечно малые при $\rho \rightarrow+0$ и получаем предел

$$
\begin{aligned}
-\int_{\theta_{H}} \cos \psi(\zeta) \sum_{\alpha, \beta=0, \pm} \overline{K_{\beta}(\zeta)} K_{\alpha}(\zeta) Q_{\rho}\left(U^{\alpha}, \partial_{\eta} U^{\beta}\right) d \zeta & \\
& =-\int_{\theta_{H}} \cos \psi(\zeta) \sum_{\alpha=0, \pm}\left|K_{\alpha}(s)\right|^{2} d \zeta
\end{aligned}
$$

Таким образом, формула Грина (43) преобразуется в равенство (14), содержащее знак плюс, но требующее понятных переобозначений. В итоге при учете (44) получаем, что $\partial_{z} u^{t r}=0$ на $\omega^{0}$, а значит, $u^{t r}=0$ всюду в $\Omega$ по теореме о единственности продолжения для ортотропной системы уравнений теории упругости (см. пояснения в [5, § 2, п. 1], а также статью [21], позволяющую расширить область применения используемых результатов из $[11$, гл. 4]).

Теорема 2. Точечный спектр оператора $\mathscr{A}_{\Gamma}$ задачи (6), (7) в случае ортотропного тела пуст, т. е. у нее нет решений из пространства Соболева $H^{1}(\Omega)^{3}$.

5. Существование захваченных волн. Задача (6), (8) требует совершенно иного подхода по следующей причине: направление оси $\eta$, «смотрящей из зоны Дирихле в зону Неймана», меняется на противоположное, а значит, первый множитель в правой части (46) принимает вид $-\cos \psi(\zeta)$, т. е. в равенство (14) прибывает знак минус, нарушающий ход рассуждений в п. 4. Убедимся в том, что при большой длине

$$
l=\min _{s \in \partial \omega} H(s)
$$

зоны Неймана у задачи (6), (8) обязательно появляется захваченная волна. Подчеркнем, что этот результат выводится при любой анизотропии упругого тела. 
Применим минимальный принцип (см., например, [4, гл. 10]) к оператору $\mathscr{A} / \Sigma$, нижняя грань спектра которого находится по формуле

$$
\inf _{u \in \mathscr{H}_{\Sigma}} \frac{\left(A D\left(\nabla_{x}\right) u, D\left(\nabla_{x}\right) u\right)_{\Omega}}{\left\|u ; L^{2}(\Omega)\right\|^{2}} .
$$

Непрерывный спектр оператора $\mathscr{A} \Sigma$ занимает луч $\left[\Lambda_{\dagger},+\infty\right)$ с положительной точкой отсечки $\Lambda_{\dagger}>0$ (см., например, $\left.[4, \S 3]\right)$, и если окажется, что величина (47) строго меньше $\Lambda_{\dagger}$, то дискретный спектр оператора $\mathscr{A} \Sigma$ непуст и $(47)-$ наименьшее собственное число.

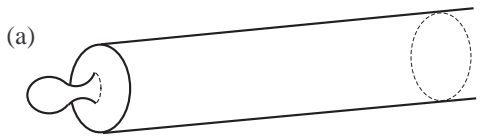

(b)

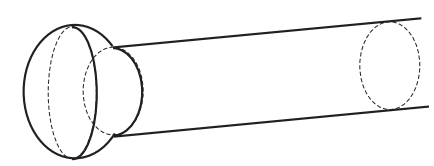

Рис. 3. Полубесконечные волноводы с «камерой» (a) и большим «резонатором» (b).

Пусть $X$ - гладкая срезающая функция,

$$
X(z)=1 \text { при } z \geq 1, \quad X(z)=0 \text { при } z \leq 0 .
$$

Положим $X_{l}(z)=X(z) X(l-z)$ и

$$
u^{l}=\left(u_{1}^{l}, u_{2}^{l}, u_{3}^{l}\right)^{\top}, \quad u_{1}^{l}(x)=X_{l}(z), u_{2}^{l}(x)=0, u_{3}^{l}(x)=-y_{1} \partial_{z} X_{l}(z) .
$$

Ясно, что $u^{l} \in \mathscr{H}_{\Sigma}$ и согласно определениям (2) и (3)

$$
\varepsilon\left(u^{l} ; x\right)=\left(0,0,-y_{1} \partial_{z}^{2} X_{l}(z), 0,0,0\right)^{\top} .
$$

Следовательно, учитывая соотношения $X_{l}(z)=1$ и $\partial_{z} X_{l}(z)=0$ при $z \in(1, l-1)$, находим, что

$$
\begin{gathered}
\left\|u^{l} ; L^{2}(\Omega)\right\|^{2} \geq c_{\omega}(l-2), c_{\omega}>0, \\
\left(A D(\nabla) u^{l}, D(\nabla) u^{l}\right)_{\Omega}=\left(A \varepsilon\left(u^{l}\right), \varepsilon\left(u^{l}\right)\right)_{\Omega} \leq 2 c_{A X} .
\end{gathered}
$$

Таким образом, дробь Рэлея из (47), вычисленная для поля смещений (48), не превосходит $c_{A X} c_{\omega}^{-1}(l-2)^{-1}$ и исчезает в пределе при $l \rightarrow+\infty$. Нужное утверждение установлено.

Теорема 3. Найдется такая величина $l_{0}$, зависящая от $\omega$ и $A$, что при $l>l_{0}$ дискретный спектр оператора $\mathscr{A}$ 乏 задачи (6), (8) анизотропной теории упругости непуст, т. е. у этой задачи есть нетривиальное решение $u^{t r}$ из пространства Соболева $H^{1}(\Omega)^{3}$.

6. Несколько замечаний. $1^{0}$. ТоРЕц. Прямой торец полубесконечного цилиндра $\Omega=\omega \times \mathbb{R}_{+}$(см. рис. 1(а)) значительно упростил проверку теоремы 2 . Утверждение об отсутствии точечного спектра в задаче (6), (7) сохраняется и для цилиндра (11) с искривленным торцом $v^{h}$ (см. рис. $\left.1(\mathrm{~b})\right)$; необходимое изменение аргументации можно найти в $[5, \S 2]$. Тот факт, что торец $\omega^{h}$ является графиком функции $h$, играет принципиальное значение, поскольку при 
наличии «камер» (рис. 3(а)) воспользоваться приемом Рэллиха не удается: проекция нормали на ось $z$ меняет знак. Наконец, теорема 2 сохраняет силу для системы (6) с условиями Дирихле на всей границе цилиндра (11) (см. [5]), но в случае появления достаточно большого «резонатора» $G \subset \mathbb{R}_{-}^{3}$ задача Дирихле в упругом волноводе $\Omega \cup \omega^{0} \cup G$ (рис. 3(b)) приобретает непустой дискретный спектр (см. доказательство в $[5, \S 3]$ и оригинальную работу [22], опирающуюся на минимальный принцип (47)).

$2^{0}$. Линия РАЗДЕЛА. Для того чтобы гарантировать положительность второго слагаемого в левой части (14), линия раздела (10) краевых условий должна быть графиком функции (иначе может быть нарушено неравенство $\cos \psi(s)>0)$. Впрочем, автор не знает, как построить примеры захваченных упругих волн при наличии «выступов» или «пятен» Дирихле (ср. рис. 4(a), (b)). С другой стороны появление «пятна» Неймана $S$ (см. рис. $4(\mathrm{~b}))$ на боковой поверхности цилиндра $\Omega$ с краевыми условиями

$$
u(x)=0, x \in\left(\partial \omega \times \mathbb{R}_{+}\right) \backslash \bar{S}, \quad D(\nu(x))^{\top} A D(\nabla) u(x)=0, x \in \omega^{0} \cup S,
$$

обязательно приводит к возникновению собственного числа в дискретном спектре задачи (6), (49) (легко проверяется опять-таки при помощи минимального принципа (47)).

(a)

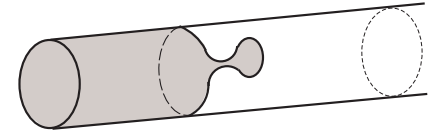

(b)

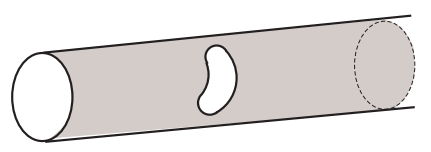

Рис. 4. Волноводы с «выступом» Дирихле (a) и«пятном» Неймана (b).

$3^{0}$. О ГлАдКости ГРАницы. Введенное требование бесконечной гладкости кривых $\partial \omega^{0}$ и $\theta_{H}$, конечно, излишне и достаточно предположить их принадлежность классу Гёльдера $C^{1, \delta}, \delta>0$. Если эти кривые теряют гладкость и становятся, например, кусочно гладкими, то точки излома дуг порождают сингулярности решений, отличающиеся от изученных в [5] и п. 4 данной статьи. Иными словами, вопрос о справедливости утверждений теоремы 2 для многогранного упругого волновода на рис. 2(b) требует анализа сингулярностей упругих полей вблизи вершин многогранников с разнотипными краевыми условиями на гранях (автор собирается закончить такое, весьма громоздкое, исследование в другой работе).

$4^{0}$. КРАТНОСТь ДИСКРеТНОГО СПЕКТРА. Прием, использованный в п. 5, малочувствителен к форме и дифференциальным свойствам границы волновода: для проверки теоремы 3 нужно лишь, чтобы полубесконечная часть $\partial \omega \times\left(H_{\infty},+\infty\right)$ боковой поверхности была зафиксирована (условия Дирихле), а длинная $(l \gg 1)$ часть $\partial \omega \times\left(H_{0}, H_{0}+l\right)$ была свободна от внешних нагрузок (условия Неймана). Более того, сечение образующей $\partial v$ последней может отличаться от $\partial \omega$ (см. тоннельную трещину на рис. $2($ a)). Наконец, заменив 
минимальный принцип максиминимальным (ср. теоремы 10.2.1, 10.2.2 в [4]) и применив асимптотическую теорию длинных (тонких после масштабирования) упругих стержней так, как это было сделано в [23, §6], можно убедиться в том, что полная кратность дискретного спектра неограниченно возрастает при $l \rightarrow+\infty$.

\section{ЛИТЕРАТУРА}

1. Лехницкий С. Г. Теория упругости анизотропного тела. М.: Наука, 1977.

2. Ладыженская О. А. Краевые задачи математической физики. М.: Наука, 1973.

3. Фикера Г. Теоремы существования в теории упругости. М.: Мир, 1974.

4. Бирман M. 3., Соломяк M. 3. Спектральная теория самосопряженных операторов в гильбертовом пространстве. Л.: Изд-во Ленингр. ун-та, 1980.

5. Назаров C. А. Упругие волны, захваченные однородным анизотропным полуцилиндром // Мат. сб. 2013. Т. 204, № 11. С. 99-130.

6. Rellich $F$. Über das asymptotische Verhalten der Lösungen von $\Delta u+\lambda u=0$ in unendlichen Gebiete // Jahresber. Dtsch. Math.-Ver. 1943. Bd 53, Heft 1. S. 57-65.

7. Nazarov $S$. A. Properties of spectra of boundary value problems in cylindrical and quasicylindrical domains // Sobolev Spaces in Mathematics. V. II (V. Maz'ya, ed.). Intern. Math. Ser. New York: Springer-Verl., 2008. V. 9. P. 261-309.

8. Nazarov S. A., Plamenevsky B. A. Elliptic problems in domains with piecewise smooth boundaries. Berlin; New York: Walter de Gruyter, 1994.

9. Назаров С. А. Трещина на стыке анизотропных тел. Сингулярности напряжений и инвариантные интегралы // Прикл. математика и механика. 1998. Т. 62, № 3. С. 489-502.

10. Назаров C. А. Трещина на стыке анизотропных тел. Сингулярности упругих полей и критерии разрушения при контакте берегов // Прикл. математика и механика. 2005. T. 69 , № 3. C. 520-532.

11. Leis R. Initial boundary value problems of mathematical physics. Stuttgart: B. G. Teubner, 1986.

12. Назаров С. А. Полиномиальное свойство самосопряженных эллиптических краевых задач и алгебраическое описание их атрибутов // Успехи мат. наук. 1999. Т. 54, № 5. С. $77-142$.

13. Кондратьев В. А., Олейник О. А. Краевые задачи для системы теории упругости в неограниченных областях. Неравенство Корна // Успехи мат. наук. 1988. Т. 43, № 5. C. $55-98$.

14. Михлин С. Г. Вариационные методы в математической физике. М.: Наука, 1970.

15. Ван Дайк М. Д. Методы возмущений в механике жидкостей. М.: Мир, 1967.

16. Ильин А. М. Согласование асимптотических разложений решений краевых задач. М.: Наука, 1989.

17. Maz'ya V., Nazarov S., Plamenevskij B. Asymptotic theory of elliptic boundary value problems in singularly perturbed domains. Basel: Birkhäuser-Verl., 2000.

18. Мазья В. Г., Пламеневский Б. А. О коэффициентах в асимптотике решений эллиптических краевых задач в области с коническими точками // Math. Nachr. 1977. V. 76, N . Р. 29-60.

19. Кондратьев B. А. О гладкости решений задачи Дирихле для эллиптического уравнения второго порядка в окрестности ребра // Дифференц. уравнения. 1970. Т. 6, № 10. C. $1831-1843$.

20. Мазья В. Г., Пламеневский Б. А. Об эллиптичности краевых задач в областях с кусочно гладкой границей // Тр. симпозиума по мех. сплошных сред и родственным пробл. анализа. Тбилиси: Мецниереба, 1973. Т. 1. С. 171-181.

21. Лангер С., Назаров С. А., Шпековиус-Нойгебауер М. Аффинные преобразования трехмерных анизотропных сред и явные формулы для фундаментальных матриц // Прикл. механика и техническая физика. 2006. Т. 47, № 2. С. 95-102.

22. Jones $D$. S. The eigenvalues of $\nabla^{2} u+\lambda u=0$ when the boundary conditions are given on semi-infinite domains // Proc. Camb. Phil. Soc. 1953. V. 49, N . P. 668-684.

23. Назаров C. А. Энергетические условия излучения Мандельштама и вектор Умова Пойнтинга в упругих волноводах // Проблемы математического анализа. Новосибирск: 
Науч. книга, 2013. Т. 72. С. 101-146.

Поступила в редакиию 13 августа 2018 г.

После доработки 13 августа 2018 г.

Принята к публикачии 18 октября 2019 г.

Назаров Сергей Александрович

Санкт-Петербургский государственный университет,

Университетская набережная, 7-9, Санкт-Петербург 199034

srgnazarov@yahoo.co.uk 